\title{
Co-existing patterns of MRI lesions were differentially associated with knee pain at rest and on joint loading: a within-person knee-matched case-controls study
}

Qiang Liư ${ }^{1,2}$, Nancy E. Lane ${ }^{3}$, David Hunter ${ }^{4}$, Dan Xing ${ }^{1}$, Zhikun Li ${ }^{1}$, Jianhao Lin ${ }^{1 *}$ (D) and Yuqing Zhang ${ }^{2 *}$

\begin{abstract}
Background: To assess the association of co-existing MRI lesions with knee pain at rest or on joint loading. Methods: We included participants from Osteoarthritis Initiative whose pain score, measured by WOMAC subscales, differed by $\geq 1$ point at rest (in bed at night, sitting/lying down) or on joint loading (walking, stairs) between two knees. Cartilage morphology, bone marrow lesions, meniscus extrusion, meniscus morphology, Hoffa's synovitis and synovitis-effusion were assessed using the compartment-specific MRI Osteoarthritis Knee Score. We performed latent class analyses to identify subgroups of co-existing MRI lesions and fitted a conditional logistic regression model to examine their associations with knee pain.

Results: Among 130 eligible participants, we identified five subgroups of knees according to patterns of co-existing MRI lesions: I. minimal lesions; II. mild lesions; II. moderate morphological lesions; IV. moderate multiple reactive lesions; and V. severe lesions. Compared with subgroup I, the odds ratios (ORs) and 95\% confidence intervals (Cl) of greater pain in bed at night were $1.6(0.3,7.2), 2.2(0.5,9.5), 6.2(1.3,29.6)$ and $11.2(2.1,59.2)$ for subgroups $\|-V_{\text {, }}$ respectively. A similar association was observed between aforementioned subgroups and pain with sitting/lying down. The ORs $(95 \% \mathrm{Cl})$ of greater pain with walking were 1.0 (reference), $1.7(0.5,6.1), 0.7(0.2,2.3), 5.0(1.4,18.6)$ and $7.9(2.0,31.5)$ for subgroup I-V, respectively. The corresponding analysis for pain on stairs showed similar results.

Conclusions: Distinct patterns of co-existing MRI lesions have different implications for the pathogenesis of osteoarthritic knee pain occurring with/without joint loading.
\end{abstract}

Keywords: MRI, Pattern, Pain, Joint loading, Knee, Osteoarthritis

\footnotetext{
* Correspondence: linjianhao@pkuph.edu.cn; yzhang108@mgh.harvard.edu

'Peking University People's Hospital, Arthritis Clinic and Research Center,

No.11 Xizhimen South Road, Xicheng District, Beijing 100044, China

${ }^{2}$ Division of Rheumatology, Allergy, and Immunology, Massachusetts General Hospital, 55 Fruit St, Boston, MA 02114, USA

Full list of author information is available at the end of the article
}

C C The Author(s). 2020 Open Access This article is licensed under a Creative Commons Attribution 4.0 International License, which permits use, sharing, adaptation, distribution and reproduction in any medium or format, as long as you give appropriate credit to the original author(s) and the source, provide a link to the Creative Commons licence, and indicate if changes were made. The images or other third party material in this article are included in the article's Creative Commons licence, unless indicated otherwise in a credit line to the material. If material is not included in the article's Creative Commons licence and your intended use is not permitted by statutory regulation or exceeds the permitted use, you will need to obtain permission directly from the copyright holder. To view a copy of this licence, visit http://creativecommons.org/licenses/by/4.0/ The Creative Commons Public Domain Dedication waiver (http://creativecommons.org/publicdomain/zero/1.0/) applies to the data made available in this article, unless otherwise stated in a credit line to the data. 


\section{Background}

Pain at rest and pain on joint loading are two different manifestations of knee pain in osteoarthritis [1-3]. Pain at rest has been a marker for more severe cases of knee $\mathrm{OA}$ and a criterion utilized for the recommendation of total knee replacement (TKR) $[4,5]$. Pain on joint loading, e.g., pain with walking, is among the most commonly reported symptoms experienced by patients with knee OA [6], and consequently contributes to physical disability [7-9]. These two types of pain are differentially associated with other clinical variables and response to treatment $[3,10,11]$. For example, neuropathic pain is more strongly associated with pain at rest than pain on joint loading among people with end-stage hip and knee OA [10]. Additionally, patients with higher knee pain at rest are more likely to have less favorable pain relief after TKR [3], whereas pain on joint loading is improved significantly compared with pain at rest among patients treated with TKR or placebo $[3,11]$. Collectively, these findings suggest that pain at rest and pain on joint loading may have different underlying mechanisms and corresponding risk factors. However, to our knowledge, despite the different clinical relevance and impact on patients, differential risk factors for these two types of osteoarthritic knee pain are poorly understood.

Knee OA is a disease of the whole joint featured by structural changes in a number of tissues including cartilage, meniscus, synovium and subchondral bone. These lesions can be identified and assessed in a compartment-specific semi-quantitative way on MRI [12]. Studies on different MRI structural lesions have primarily focused on individual MRI lesions and knee OA outcomes [13]. Focusing on a single MRI feature or structural lesion may be overly simplistic as it does not give a comprehensive picture of the relation between MRI lesions and pain in this whole joint disease. For example, articular cartilage is incapable of directly generating pain because it is aneural. However, cartilage defects, in isolation from other tissues, have been reported to be associated with knee pain in OA [14, 15]. Such an association may limit insights into the pathogenesis of osteoarthritic knee pain.

Studying the relation of multiple MRI lesions to knee pain in OA is methodologically challenging. First, many factors that account for an individual's pain response, such as central hypersensitivity, are either not collected or not controlled for in most observational studies [16]; thus the validity of study findings related to an individual's pain could be affected by residual confounding. A within-person knee-matched case-control study design has been proposed to eliminate person-level confounders and improve the validity of study findings $[17,18]$. Second, to include all MRI lesions into a multivariable regression model and obtain their "independent" effects may be problematic in the absence of knowledge regarding the temporal sequence among the occurrences of MRI lesions. Some effect estimates represent the total effect, and others direct effects, according to the chronology of the occurrence $[19,20]$. An alternative strategy is to identify the patterns of co-existing MRI lesions and examine their relation to knee pain in OA [21], which is attractive because model building with many highly correlated predictors can be otherwise nearly impossible. To date, there are no published studies that have utilized these approaches to examine the associations of multiple MRI lesions with subtypes of osteoarthritic knee pain. Therefore, we conducted a within-person knee-matched case-control study to determine if distinct patterns of co-existing MRI lesions have differential associations with knee pain at rest and on joint loading using data collected from the Osteoarthritis Initiative (OAI).

\section{Methods}

Study sample

The OAI is a longitudinal cohort study of participants with or at high risk of knee OA. At baseline, the OAI cohort included 4796 subjects aged $45-79$ years who were recruited from four sites, Columbus, Ohio, Providence, Rhode Island, Baltimore, Maryland and Pittsburgh, Pennsylvania. Annual assessments included the questionnaires, clinical examination and imaging.

\section{Knee pain at rest and knee pain on joint loading}

We used the knee-specific Western Ontario and McMaster Universities Osteoarthritis Index (WOMAC) pain sub-scales $(0-5)$ to define knee pain at rest and on joint loading. The WOMAC pain sub-scales measure the severity of pain occurring in five scenarios during the past 7 days. For the current study, we used the items of pain with sitting/lying down and pain in bed at night as two separate measures of knee pain at rest, and those of pain with walking and pain on stairs as measures of knee pain on joint loading.

\section{MRI assessments}

MRI readings of structural lesions of knee OA, including cartilage morphology (CartM), bone marrow lesion (BML), synovitis-effusion, Hoffa's synovitis (HFS), meniscus morphology (MM) and meniscus extrusion (MExt), were performed using the compartment-specific semi-quantitative MRI Osteoarthritis Knee Score (MOAKS) in the OAI [12]. Scores for BML and CartM were applied in subregions for each lesion in both the tibiofemoral joint (TFJ) and patellofemoral joint (PFJ), denoted by TFJ-BML, PFJ-BML, TFJ-CartM and PFJCartM, respectively. We only pooled data from the knee MRI scans that were read by Boston Imaging Core Laboratory group. We restricted our analyses to those who had MRI readings for both knees in one visit. 


\section{Statistical analysis}

The outcome of interest is a greater score of each of WOMAC pain sub-scales respectively. We identified a matched pair of case and control knees within one participant if his/her two knees differed by $\geq 1$ point in at least one score of knee pain at rest or on joint loading (Fig. 1). Given that the comparison is between two knees within one person, we consider a discordance of $\geq 1$ point as a meaningful difference which is consistent with the magnitude of acute pain flare evoked by an exercise session [22]. Participants were included when their knees formed a matched pair in the earliest visit between baseline and the 48-month visit. Each participant was included only once.

Scores for MRI features were from the same visit when a pair of knees was included. We used the worst CartM score from among 12 subregions in the TFJ (medial and lateral trochlea, medial and lateral central femur, medial and lateral posterior femur; medial anterior tibia, medial central tibia, medial posterior tibia, lateral anterior tibia, lateral central tibia and lateral posterior tibia) and among 2 subregions in the PFJ (medial and lateral patella on the axial view) to represent the severity of cartilage damage in each of the tibiofemoral and patellofemoral joints, respectively. The worst BML score for size from among the aforementioned 12 subregions in the TFJ and among the aforementioned 2 subregions in the PFJ was used to represent the severity of BML in each joint, respectively. The worst MM score from among 6 subregions (medial anterior, body, and posterior; lateral anterior, body, and posterior) and the worst MExt score from among 2 areas (medial and lateral) in 2 views (coronal and sagittal) were used to represent the severity of these lesions in the whole knee.

We performed latent class analysis (LCA) using "gsem" STATA procedure that fits a generalized structural equation model with categorical latent variables to identify subgroups representing distinct patterns of co-existing MRI lesions based on the prevalence and severity of CartM and BML in the TFJ and PFJ, as well as EFF, HFS, MM and MExt in the whole knee. If the proportion of knees with a score level of an MRI lesion was less than $3 \%$, we collapsed those knees to the adjacent lower score level to avoid unstable estimations due to sparse data. We fitted the LCA models with 2-6 subgroups and chose the model according to the following criteria: 1) to have a meaningful clinical relevance; 2) the lowest Akaike information criterion or Schwarz's Bayesian information criterion to identify the best model fit [23]; 3) to have sufficient numbers of knees ( $\geq 10 \%$ of the sample) in each subgroup (Table 1). We assigned each knee to the subgroup with the maximum posterior probability generated from LCA model. We then fitted a conditional logistic regression model to deal with the dependence of two knees within one subject in a separate analysis of pain at rest and pain on joint loading. We performed test of homogeneity of odds ratios (ORs) using "tabodds" STATA procedure. Subjects who had a TKR or had missing values for variables of interest in either knee were excluded. All statistical analyses were performed using Stata/SE 15.1 (StataCorp, Texas, USA).

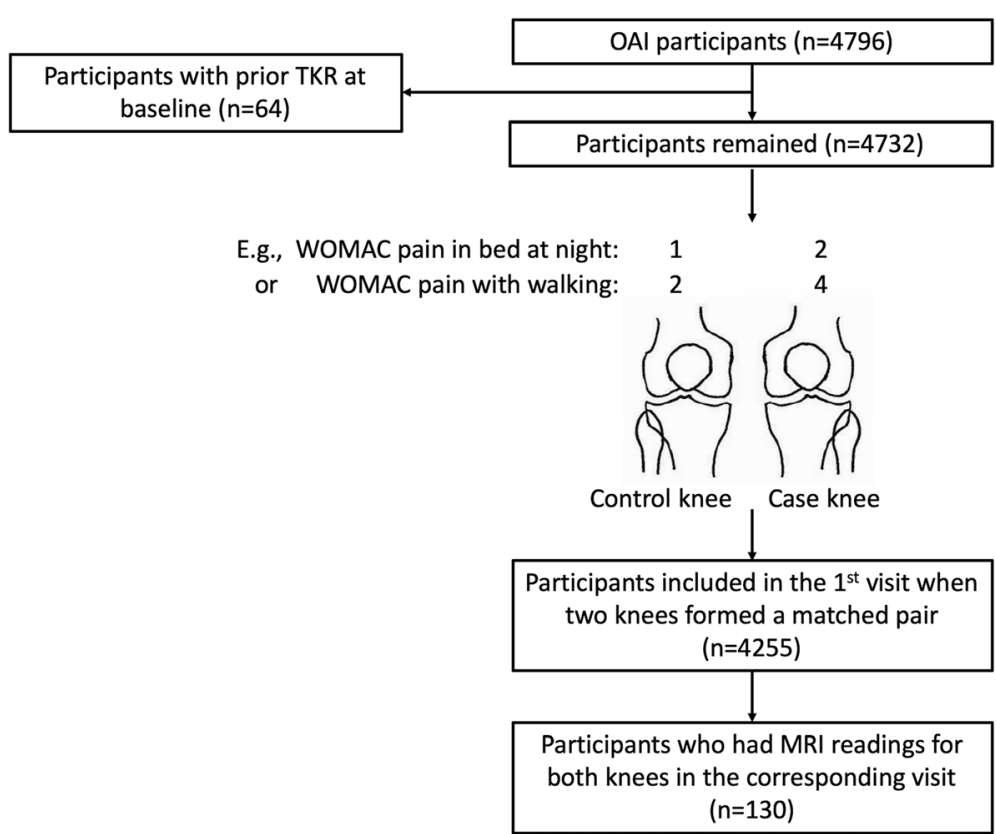

Fig. 1 Osteoarthritis Initiative participants included in final analyses until 48-month follow-up. OAI = Osteoarthritis Initiative, TKR=Total knee replacement, WOMAC = the Western Ontario and McMaster Universities Osteoarthritis Index, E.g. = For example 
Table 1 Goodness-of-fit statistics for latent class models

\begin{tabular}{llll}
\hline No. subgroups & AIC & BIC & Average posterior probability \\
\hline 2 & 4955.023 & 5044.04 & 96.1 \\
3 & 4827.233 & 4948.296 & 95.2 \\
4 & 4778.447 & 4931.556 & 95.3 \\
5 & 485.4094 & 542.3803 & 96.0 \\
6 & 953.0936 & 1010.065 & 96.6
\end{tabular}

AIC Akaike information criterion, BIC Schwarz's Bayesian information criterion

\section{Results}

Among 130 eligible participants whose MRI-lesion assessments were available, $60 \%$ were women, their mean age was 63.8 years, more than $80 \%$ of participants were White, and the mean BMI was $29.6 \mathrm{~kg} /$ $\mathrm{m}^{2}$. Among 260 knees from 130 eligible participants, the prevalence of Kellgren/Lawrence grade 0, 1, 2, 3 and 4 was $15.8,28.0,19.7,24.8$ and $11.8 \%$, respectively.

\section{Patterns of co-existing MRI lesions}

We identified five subgroups of knees based on the patterns of co-existing MRI lesions (Table 1): I $(n=36$, $13.9 \%)$, II $(n=30,11.5 \%)$, III $(n=76,29.2 \%)$, IV $(n=65$, $25.0 \%)$ and $\mathrm{V}(n=53,20.4 \%)$ (Fig. 2$)$. The average posterior probability of membership was 1.00 for subgroups I, II and V, 0.93 for subgroup III and 0.92 for subgroup $\mathrm{IV}$, respectively, suggesting that subgroup assignment was acceptably unambiguous.

The patterns of co-existing MRI lesions are depicted in Table 2 and Fig. 2. In knees of subgroup I, there was no TFJ cartilage damage, whereas all knees of subgroup II had mild TFJ-CartM. All knees of subgroup III and IV had moderate TFJ-CartM, while the prevalence of severe TFJ-CartM was $100 \%$ in subgroup $\mathrm{V}$. The prevalence and severity of $\mathrm{MM}$ and MExt also increased from subgroup I to subgroup V. Compared with subgroup III, subgroup IV was featured by a higher prevalence and greater severity of EFF, HFS and TFJ-BML. EFF, HFS and TFJ-BML

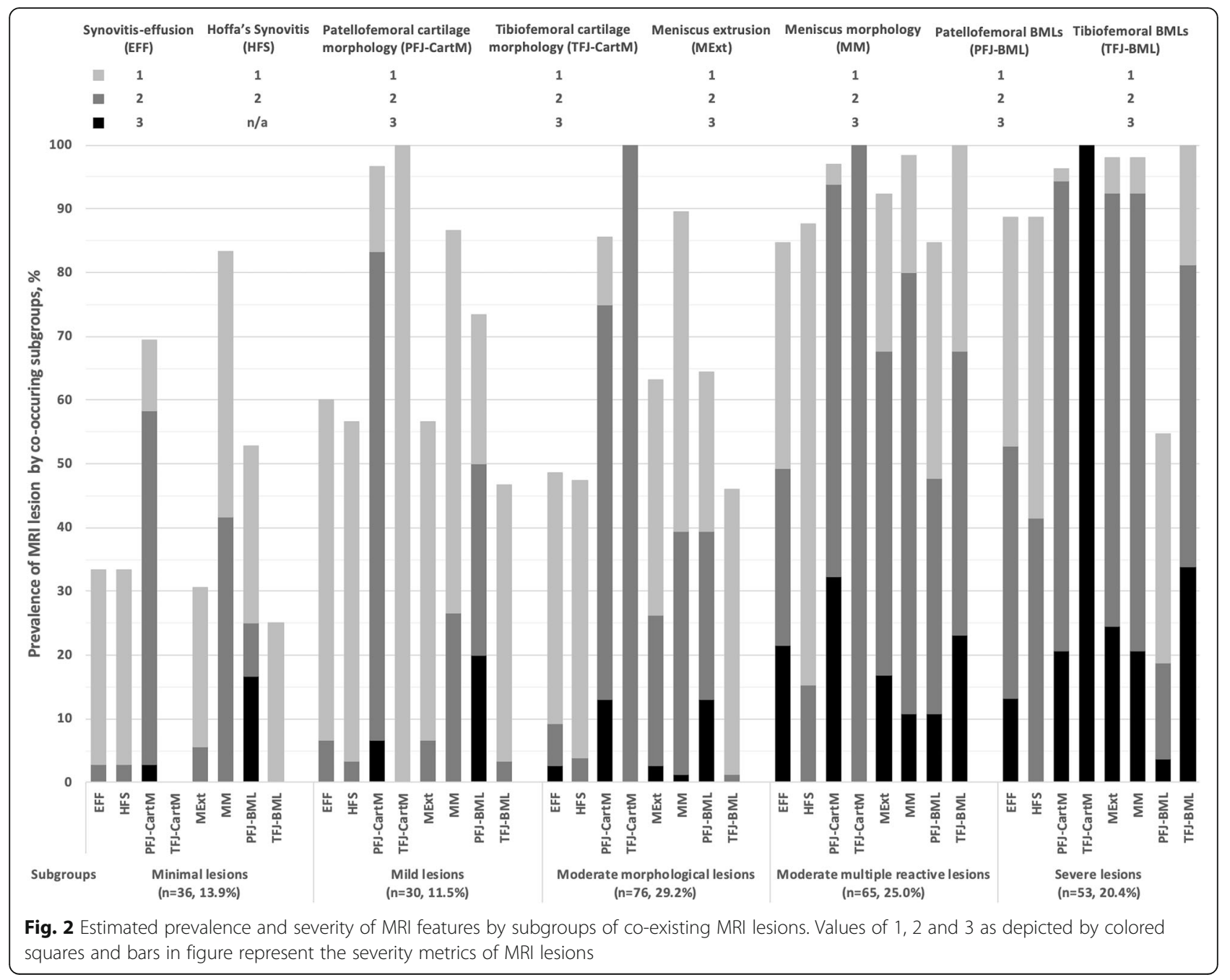


Table 2 Prevalence of MRI lesions according to latent subgroups of knees ${ }^{a}$

\begin{tabular}{|c|c|c|c|c|c|c|}
\hline MRI features & Score & $\begin{array}{l}\text { Subgroup I: } \\
\text { Minimal lesions }\end{array}$ & $\begin{array}{l}\text { Subgroup II: } \\
\text { Mild lesions }\end{array}$ & $\begin{array}{l}\text { Subgroup III: Moderate } \\
\text { morphological lesions }\end{array}$ & $\begin{array}{l}\text { Subgroup IV: Moderate } \\
\text { multiple reactive lesions }\end{array}$ & $\begin{array}{l}\text { Subgroup V: } \\
\text { Severe lesions }\end{array}$ \\
\hline \multirow[t]{4}{*}{ Synovitis-Effusion } & 0 & 66.7 & 40.0 & 51.3 & 15.4 & 11.3 \\
\hline & 1 & 30.6 & 53.3 & 39.5 & 35.4 & 35.9 \\
\hline & 2 & 2.8 & 6.7 & 6.6 & 27.7 & 39.6 \\
\hline & 3 & 0.0 & 0.0 & 2.6 & 21.5 & 13.2 \\
\hline \multirow[t]{3}{*}{ Hoffa's Synovitis } & 0 & 66.7 & 43.3 & 52.6 & 12.3 & 11.3 \\
\hline & 1 & 30.6 & 53.3 & 43.4 & 72.3 & 47.2 \\
\hline & 2 & 2.8 & 3.3 & 4.0 & 15.4 & 41.5 \\
\hline \multirow{4}{*}{$\begin{array}{l}\text { Patellofemoral } \\
\text { cartilage morphology }\end{array}$} & 0 & 30.6 & 3.3 & 14.5 & 3.1 & 3.8 \\
\hline & 1 & 11.1 & 13.3 & 10.5 & 3.1 & 1.9 \\
\hline & 2 & 55.6 & 76.7 & 61.8 & 61.5 & 73.6 \\
\hline & 3 & 2.8 & 6.7 & 13.2 & 32.3 & 20.8 \\
\hline \multirow{4}{*}{$\begin{array}{l}\text { Tibiofemoral cartilage } \\
\text { morphology }\end{array}$} & 0 & 100.0 & 0.0 & 0.0 & 0.0 & 0.0 \\
\hline & 1 & 0.0 & 100.0 & 0.0 & 0.0 & 0.0 \\
\hline & 2 & 0.0 & 0.0 & 100.0 & 100.0 & 0.0 \\
\hline & 3 & 0.0 & 0.0 & 0.0 & 0.0 & 100.0 \\
\hline \multirow[t]{4}{*}{ Meniscus extrusion } & 0 & 69.4 & 43.3 & 36.8 & 7.7 & 1.9 \\
\hline & 1 & 25.0 & 50.0 & 36.8 & 24.6 & 5.7 \\
\hline & 2 & 5.6 & 6.7 & 23.7 & 50.8 & 67.9 \\
\hline & 3 & 0.0 & 0.0 & 2.6 & 16.9 & 24.5 \\
\hline \multirow{4}{*}{$\begin{array}{l}\text { Meniscus } \\
\text { morphology }\end{array}$} & 0 & 16.7 & 13.3 & 10.5 & 1.5 & 1.9 \\
\hline & 1 & 41.7 & 60.0 & 50.0 & 18.5 & 5.7 \\
\hline & 2 & 41.7 & 26.7 & 38.2 & 69.2 & 71.7 \\
\hline & 3 & 0.0 & 0.0 & 1.3 & 10.8 & 20.8 \\
\hline \multirow{4}{*}{$\begin{array}{l}\text { Patellofemoral bone } \\
\text { marrow lesions }\end{array}$} & 0 & 47.2 & 26.7 & 35.5 & 15.4 & 45.3 \\
\hline & 1 & 27.8 & 23.3 & 25.0 & 36.9 & 35.9 \\
\hline & 2 & 8.3 & 30.0 & 26.3 & 36.9 & 15.1 \\
\hline & 3 & 16.7 & 20.0 & 13.2 & 10.8 & 3.8 \\
\hline \multirow{4}{*}{$\begin{array}{l}\text { Tibiofemoral bone } \\
\text { marrow lesion }\end{array}$} & 0 & 75.0 & 53.3 & 54.0 & 0.0 & 0.0 \\
\hline & 1 & 25.0 & 43.3 & 44.7 & 32.3 & 18.9 \\
\hline & 2 & 0.0 & 3.3 & 1.3 & 44.6 & 47.2 \\
\hline & 3 & 0.0 & 0.0 & 0.0 & 23.1 & 34.0 \\
\hline
\end{tabular}

${ }^{a}$ Values are displayed in percentage

often coexisted and their prevalence and severity were highly correlated. The prevalence and severity of PFJCartM and PFJ-BML was not correlated with that of TFJ-CartM. For example, although knees of subgroup $\mathrm{V}$ had greater TFJ-CartM than knees of subgroup IV, the prevalence and severity of PFJ-CartM and PFJBML was lower in subgroup V. Based on the differences in prevalence and severity of MRI lesions across subgroups, we labeled them as I: minimal lesions, II: mild lesions, III: moderate morphological lesions, IV: moderate multiple reactive lesions and $\mathrm{V}$ : severe lesions.

\section{Subgroups of co-existing MRI lesions and knee pain at} rest

The risk of having greater knee pain at rest increased from subgroup II to subgroup V compared with subgroup I (Table 3). The ORs and 95\% confidence intervals (CI) of greater pain in bed at night were 1.0 (reference), $1.6(0.3,7.2), 2.2(0.5,9.5), 6.2(1.3,29.6)$ and $11.2(2.1$, 59.2) from subgroup I to subgroup $V$, respectively (P for test of homogeneity $=0.056$ ), indicating that risk of pain at rest varied among the different subgroups. Similar results were observed when knee pain at rest was measured using WOMAC subscale of pain that occurs when 
Table 3 Subgroups of co-existing MRI lesions and knee pain at rest

\begin{tabular}{|c|c|c|c|c|c|c|}
\hline \multirow[b]{3}{*}{ Subgroups } & \multicolumn{6}{|c|}{ Knee pain at rest } \\
\hline & \multicolumn{3}{|c|}{ Pain in bed at night } & \multicolumn{3}{|c|}{ Pain with sitting/lying down } \\
\hline & Control knee & Case knee & OR $(95 \% \mathrm{Cl})$ & Control knee & Case knee & OR $(95 \% \mathrm{Cl})$ \\
\hline I. Minimal lesions & 13 & 7 & 1.0 (Reference) & 16 & 8 & 1.0 (Reference) \\
\hline II. Mild lesions & 14 & 8 & $1.6(0.3,7.3)$ & 14 & 4 & $1.1(0.2,5.6)$ \\
\hline III. Moderate morphological lesions & 29 & 22 & $2.2(0.5,9.5)$ & 31 & 20 & $3.1(0.7,14.4)$ \\
\hline IV. Moderate multiple reactive lesions & 22 & 28 & $6.2(1.3,29.6)$ & 20 & 31 & $14.4(2.5,81.7)$ \\
\hline \multirow[t]{2}{*}{ V. Severe lesions } & 14 & 27 & $11.2(2.1,59.2)$ & 13 & 31 & $28.9(4.5,184.0)$ \\
\hline & \multicolumn{3}{|c|}{$P$ for test of homogeneity $=0.056$} & \multicolumn{3}{|c|}{$P$ for test of homogeneity $<0.001$} \\
\hline
\end{tabular}

OR Odds ratio, $\mathrm{Cl}$ Confidence interval

Figures are numbers unless stated otherwise

subjects were sitting/lying down. The corresponding ORs were 1.0 (reference), $1.0(0.2,5.6), 3.1$ (0.7, 14.4), $14.4(2.5,81.7)$ and $28.9(4.5,184.0)$, respectively $(P$ for test of homogeneity < 0.001) (Table 3).

\section{Subgroups of co-existing MRI lesions and knee pain on joint loading}

Knees of subgroup IV and V had a higher risk of greater knee pain with walking compared with subgroup I (Table 4). The ORs of greater pain with walking were 1.0 (reference), $1.7(0.5,6.1), 0.7(0.2,2.3), 5.0(1.4,18.6)$ and $7.8(2.0,31.5)$ from subgroup I to subgroup V, respectively ( $\mathrm{P}$ for test of homogeneity $=0.001$ ), suggesting that risk of pain with walk varied statistically significantly among five subgroups. Using WOMAC item for pain on stairs as one other measure of knee pain on joint loading, the corresponding ORs were 1.0 (reference), 0.9 (0.3, 3.3), $0.8(0.3,2.5), 2.4(0.7,7.4)$ and $6.6(1.8,23.9)$, respectively ( $P$ for test of homogeneity $=0.003$ ) (Table 4$)$.

\section{Discussion}

Among the OAI participants with or at high risk of knee OA, we identified five distinct patterns of co-existing MRI lesions that were differentially associated with subtypes of knee pain in OA. These findings not only allow a glimpse of distinct clinically relevant pathways leading to knee pain, but also provide insights into the pathogenesis of knee pain in OA.

When we compared subgroup III (moderate morphological lesions) with subgroup I (minimal lesions), the results suggested that morphologic lesions of cartilage and meniscus were not significantly associated with knee pain at rest or knee pain on joint loading. Aneural hyaline articular cartilage and the meniscus do not generate pain directly, or appear to have a limited role in the pathogenesis of pain. Although there is data that vascular penetration and nerve growth after a meniscal tear may be a source of pain [24], osteoarthritic knees with a meniscal tear are not more painful than those without a tear [25]. Therefore, meniscal tears are an unlikely immediate source of knee pain in OA [26], underlying the large body of evidence that arthroscopic surgery targetting meniscus was no better than conservative interventions such as exercise therapy in pain relief [27].

BMLs reflect increased focal loading in the subchondral bone [28]. Synovitis-effusion is thought to be an inflammatory reaction that could be triggered by structural damage. Consistent with the published study [21], we found that synovitis-effusion and TFJ-BML often coexisted, especially with TFJ-CartM. Meanwhile, the severity of these lesions correlated well in subgroups. In the current study, the difference between subgroup

Table 4 Subgroups of co-existing MRI lesions and knee pain on joint loading

\begin{tabular}{|c|c|c|c|c|c|c|}
\hline \multirow[b]{3}{*}{ Subgroups } & \multicolumn{6}{|c|}{ Knee pain on joint loading } \\
\hline & \multicolumn{3}{|c|}{ Pain with walking } & \multicolumn{3}{|c|}{ Pain on stairs } \\
\hline & Control knee & Case knee & OR $(95 \% \mathrm{Cl})$ & Control knee & Case knee & OR $(95 \% \mathrm{Cl})$ \\
\hline I. Minimal lesions & 16 & 11 & 1.0 (Reference) & 19 & 15 & 1.0 (Reference) \\
\hline II. Mild lesions & 15 & 14 & $1.7(0.5,6.1)$ & 16 & 10 & $0.9(0.3,3.3)$ \\
\hline III. Moderate morphological lesions & 45 & 21 & $0.7(0.2,2.3)$ & 44 & 27 & $0.8(0.3,2.5)$ \\
\hline IV. Moderate multiple reactive lesions & 24 & 37 & $5.0(1.4,18.6)$ & 30 & 34 & $2.4(0.7,7.4)$ \\
\hline \multirow[t]{2}{*}{ V. Severe lesions } & 16 & 33 & $7.8(2.0,31.5)$ & 15 & 38 & $6.6(1.8,23.9)$ \\
\hline & \multicolumn{3}{|c|}{$P$ for test of homogeneity $=0.001$} & \multicolumn{3}{|c|}{$P$ for test of homogeneity $=0.003$} \\
\hline
\end{tabular}

OR Odds ratio, $\mathrm{Cl}$ Confidence interval

Figures are numbers unless stated otherwise 
III (moderate morphological lesions) and subgroup IV (moderate multiple reactive lesions) that have the same level of TFJ-CartM mostly lies in the prevalence and severity of EFF, HFS and TFJ-BML. Knees of subgroup IV had a higher risk of greater knee pain at rest and on joint loading than knees of subgroup III, supporting other studies that have reported that synovitis-effusion and TFJ-BML may be the major sources of knee pain in OA $[18,29]$. Given that pain is the key reason for seeking medical care [30], these lesions should be considered as clinically relevant markers of symtomatic knee OA. A recent study showed that improvement of synovitis on MRI following a transcatheter arterial embolization was associated with a significant reduction in WOMAC pain among patients with mild to moderate radiographic knee OA [31]. Studies have also shown that BMLs can be reduced by zoledronic acid or prostacyclin analogue iloprost $[32,33]$. Our findings highlight the importance of modifying either synovitis-effusion or BMLs to reduce knee pain and consequent pain-related disability.

The role of PFJ in the surgical treatment of knee OA remains controversial [34-37]. For example, there is evidence that preoperative PFJ OA or anterior knee pain does not compromise the outcome or survival of a medial unicompartmental knee replacement [34, 35]. Additionally, at the time of a TKR, patellar retention was not statistically significantly associated with the risk of incident postoperative anterior knee pain, compared with patellar resurfacing for patients receiving TKR [36]. Based on latent class analysis, we identified the coexisting pattern of BMLs and CartM that are located separately in PFJ and TFJ, allowing us to examine their differential contributions to osteoarthritic knee pain. We found that knees with greater TFJ-CartM could have lower prevalence and severity of PFJ-CartM and PFJBML (e.g., subgroup V versus subgroup IV, or subgroup III versus subgroup II). In contrast, subgroup V (severe lesions) had larger ORs of both knee pain at rest and on joint loading than subgroup IV (moderate multiple reactive lesions), suggesting that lesions in PFJ were not significantly associated with subtypes of knee pain (e.g., knee pain on stairs). Our findings, if confirmed, are informative for the management of patella during surgical treatment from the perspective of structural pathogenic source of pain.

In contrast to knee pain at rest that represents pain occurring without mechanical stimuli, knee pain on joint loading is a symptom in response to localized stress. Interestingly, joint loading can act as either a source of pain or a remedy for pain among individuals with knee OA [38]. Animal studies showed that joint loading may attenuate structural damage in $\mathrm{OA}$, depending on the amount and frequency $[39,40]$. Meanwhile, there is evidence from human studies indicates that exercise, such as land-based training, is effective in the management knee OA $[41,42]$. In the present study, we observed larger and more skewed ORs for pain at rest than for pain on joint loading. When we compared the subgroup III (moderate morphological lesions) with the subgroup I (minimal lesions), the ORs of knee pain on joint loading were less than 1.0 (i.e., 0.7 for pain with walking and 0.8 for pain on stairs) whereas the ORs of knee pain at rest were over 2.0 (i.e., 2.2 for pain in bed at night and 3.1 for pain with sitting/lying down). Collectively, these findings may add additional evidence that mechanical loading may attenuate knee pain for patients with symptomatic knee OA. It should be noted that there are other explanations. For example, various methods of distraction potentially including physical activity can be used as a modifying tactic to reduce pain [43].

Our study has several strengths. We assessed knee pain at rest and knee pain on joint loading using four items of WOMAC pain sub-scale, allowing us to separately look at knee pain in response to different pathogenic sources. Since our study design allowed the assessment of the pain difference in two knees within the same person over a short period of time, we believe that participants should be able to tell a one-point difference. Moreover, in the absence of knowledge of the temporal sequence of MRI lesions, we fitted latent class analysis to identify subgroups representing distinct patterns of co-existing MRI lesions. Although our study is subjective to limitations of a casecontrol study such a selection bias, as an advance over previous studies $[44,45]$, we were able to examine the differential association of multiple MRI lesions localized in different compartments with knee pain occurring with or without joint loading. Finally, person-level confounders were eliminated using a within-person knee-matched case-control study design; thus, the validity of the study findings was improved.

Limitations of the present study should be noted. First, although we identified patterns of co-existing MRI lesions, we still cannot tell the temporal sequence of occurrence of these MRI lesions. For example, TFJ-BML often coexisted with synovitis-effusion in subgroups [21], making it difficult to sort out their differential contributions to knee pain. Second, uncertainty in subgroup membership assignment might lead to potentially biased effect estimate, albeit the maximum-probability approach. However, the lowest average posterior probability of membership was above 0.92 among all subgroups, suggesting that subgroup assignment was robust. Third, each WOMAC pain subscale item (0-5) that we used to identify case and control knees has not been validated separately from the pain subscale as a whole. Forth, we used the difference of $\geq 1$ point for both pain at rest and pain on joint loading regardless of potentially different variance in the score for each item [46]. Finally, because 
we adopted a within-person case-control study design, we were not able to examine the association of personlevel factors, such as central hypersensitivity and coping strategy, with knee pain in OA.

\section{Conclusions}

In conclusion, co-existing patterns of MRI lesions were identified and differentially associated with knee pain at rest and on joint loading in OA. Morphological lesions of cartilage and meniscus were not statistically significantly associated with pain. Synovitis-effusion and TJF-BML were highly correlated and appeared to be the major sources of pain. PFJ lesions had a limited role in the pathogenesis of osteoarthritic knee pain. These findings are informative for optimizing a treatment strategy targeting the pathogenic sources of osteoarthritic knee pain.

\section{Abbreviations \\ OA: Osteoarthritis; TKR: Total knee replacement; OAl: The Osteoarthritis Initiative; WOMAC: Western Ontario and McMaster Universities Osteoarthritis Index; AIC: Akaike information criterion; BIC: Schwarz's Bayesian information criterion; CartM: Cartilage morphology; BML: Bone marrow lesion; EFF: Synovitis-effusion; HFS: Hoffa's synovitis; MM: Meniscus morphology; MExt: Meniscus extrusion; MOAKS: MRI Osteoarthritis Knee Score; TFJ: Tibiofemoral joint; PFJ: Patellofemoral joint; LCA: Latent class analysis; ORs: Odds ratios; Cl: Confidence interval}

\section{Acknowledgements}

We would like to thank the OAI participants, OAI investigators and OAI Clinical Center's staff for generating this publicly available data set.

\section{Authors' contributions}

$\mathrm{QL}$ and $\mathrm{DX}$ analyzed the data. QL, NL, DH, JL and $\mathrm{YZ}$ were major contributors in writing the manuscript. All authors interpreted the data and critically reviewed and revised the article for important intellectual content. All authors read and approved the final manuscript.

\section{Funding}

This work was supported by Osteoarthritis Research Society International [Collaborative Scholarship 2017 to QL]; Beijing Joint Care Foundation [Young Investigator Scholarship 2017 to QL] and the National Natural Science Foundation of China [81902247 to QL]. The Osteoarthritis Initiative is a public-private partnership comprised of five National Institutes of Health (NIH) contracts [N01-AR-2-2258; N01-AR-2-2259; N01-AR-2-2260; N01-AR-22261 and N01-AR-2-2262] funded by the NIH and conducted by the OAI Study Investigators. Private sector funding for the OAl is managed by the Foundation for the $\mathrm{NIH}$. The funding sources had no role in the design of the study and collection, analysis, and interpretation of data and in writing the manuscript.

\section{Availability of data and materials}

The datasets generated and/or analysed during the current study are available in the OAI repository, https://nda.nih.gov/oai.

\section{Ethics approval and consent to participate}

The Institutional Review Board for the University of California (San Francisco) approved the study, and written informed consent was obtained from all participants.

\section{Consent for publication}

Not Applicable.

\section{Competing interests}

The authors declare that they have no competing interests.

\section{Author details}

${ }^{1}$ Peking University People's Hospital, Arthritis Clinic and Research Center, No.11 Xizhimen South Road, Xicheng District, Beijing 100044, China. Division of Rheumatology, Allergy, and Immunology, Massachusetts General Hospital, 55 Fruit St, Boston, MA 02114, USA. ${ }^{3}$ Center for Musculoskeletal Health, University of California, Davis School of Medicine, Sacramento, USA. ${ }^{4}$ Institute of Bone and Joint Research, The Kolling Institute, Sydney Medical School, The University of Sydney, Sydney, Australia.

Received: 2 June 2020 Accepted: 29 September 2020

Published online: 06 October 2020

\section{References}

1. Neogi T, Frey-Law L, Scholz J, Niu J, Arendt-Nielsen L, Woolf C, et al. Sensitivity and sensitisation in relation to pain severity in knee osteoarthritis: trait or state? Ann Rheum Dis. 2015;74:682-8.

2. Creamer $P$, Lethbridge-Cejku M, Hochberg MC. Determinants of pain severity in knee osteoarthritis: effect of demographic and psychosocial variables using 3 pain measures. J Rheumatol. 1999;26:1785-92.

3. Lundblad $H$, Kreicbergs $A$, Jansson KA. Prediction of persistent pain after total knee replacement for osteoarthritis. J Bone Joint Surg (Br). 2008;90:166-71.

4. Naylor CD, Williams JI. Primary hip and knee replacement surgery: Ontario criteria for case selection and surgical priority. Qual Health Care. 1996;5:20-30.

5. Mancuso CA, Ranawat CS, Esdaile JM, Johanson NA, Charlson ME Indications for total hip and total knee arthroplasties. Results of orthopaedic surveys. J Arthroplast. 1996:11:34-46.

6. Murphy SL, Lyden AK, Kratz AL, Fritz H, Williams DA, Clauw DJ, et al. Characterizing pain flares from the perspective of individuals with symptomatic knee osteoarthritis. Arthritis Care Res (Hoboken). 2015;67:1 103-11.

7. Wilcox S, Der Ananian C, Abbott J, Vrazel J, Ramsey C, Sharpe PA, et al. Perceived exercise barriers, enablers, and benefits among exercising and nonexercising adults with arthritis: results from a qualitative study. Arthritis Rheum. 2006;55:616-27.

8. Somers TJ, Keefe FJ, Pells JJ, Dixon KE, Waters SJ, Riordan PA, et al. Pain catastrophizing and pain-related fear in osteoarthritis patients: relationships to pain and disability. J Pain Symptom Manag. 2009;37:863-72.

9. Hawker GA. Experiencing painful osteoarthritis: what have we learned from listening? Curr Opin Rheumatol. 2009;21:507-12.

10. Power JD, Perruccio AV, Gandhi R, Veillette C, Davey JR, Syed K, et al. Neuropathic pain in end-stage hip and knee osteoarthritis: differential associations with patient-reported pain at rest and pain on activity. Osteoarthr Cartil. 2018;26:363-9.

11. Bihlet AR, Byrjalsen I, Bay-Jensen AC, Andersen JR, Christiansen C, Riis BJ, et al. Identification of pain categories associated with change in pain in patients receiving placebo: data from two phase 3 randomized clinical trials in symptomatic knee osteoarthritis. BMC Musculoskelet Disord. 2018:19:17.

12. Hunter DJ, Guermazi A, Lo GH, Grainger AJ, Conaghan PG, Boudreau RM, et al. Evolution of semi-quantitative whole joint assessment of knee OA: MOAKS (MRI osteoarthritis knee score). Osteoarthr Cartil. 2011;19:990-1002.

13. Hunter DJ, Guermazi A, Roemer F, Zhang Y, Neogi T. Structural correlates of pain in joints with osteoarthritis. Osteoarthr Cartil. 2013;21:1170-8.

14. Sowers MF, Hayes C, Jamadar D, Capul D, Lachance $L$, Jannausch M, et al. Magnetic resonance-detected subchondral bone marrow and cartilage defect characteristics associated with pain and X-ray-defined knee osteoarthritis. Osteoarthr Cartil. 2003;11:387-93.

15. Wluka AE, Wolfe R, Stuckey S, Cicuttini FM. How does tibial cartilage volume relate to symptoms in subjects with knee osteoarthritis? Ann Rheum Dis. 2004;63:264-8.

16. Neogi T. The epidemiology and impact of pain in osteoarthritis. Osteoarthr Cartil. 2013;21:1145-53.

17. Neogi T, Felson D, Niu J, Nevitt M, Lewis CE, Aliabadi P, et al. Association between radiographic features of knee osteoarthritis and pain: results from two cohort studies. BMJ. 2009;339:b2844.

18. Zhang Y, Nevitt M, Niu J, Lewis C, Torner J, Guermazi A, et al. Fluctuation of knee pain and changes in bone marrow lesions, effusions, and synovitis on magnetic resonance imaging. Arthritis Rheum. 2011;63:691-9.

19. Westreich D, Greenland S. The table 2 fallacy: presenting and interpreting confounder and modifier coefficients. Am J Epidemiol. 2013;177:292-8.

20. Zhang Y, Neogi T, Hunter D, Roemer F, Niu J. What effect is really being measured? An alternative explanation of paradoxical phenomena in studies of osteoarthritis progression. Arthritis Care Res (Hoboken). 2014;66:658-61. 
21. Niu J, Felson DT, Neogi T, Nevitt MC, Guermazi A, Roemer F, et al. Patterns of coexisting lesions detected on magnetic resonance imaging and relationship to incident knee osteoarthritis: the multicenter osteoarthritis study. Arthritis Rheum. 2015;67:3158-65

22. Sandal LF, Roos EM, Bogesvang SJ, Thorlund JB. Pain trajectory and exerciseinduced pain flares during 8 weeks of neuromuscular exercise in individuals with knee and hip pain. Osteoarthr Cartil. 2016;24:589-92.

23. Sclove SLJP. Application of model-selection criteria to some problems in multivariate analysis, vol. 52; 1987. p. 333-43.

24. Ashraf S, Wibberley H, Mapp PI, Hill R, Wilson D, Walsh DA. Increased vascular penetration and nerve growth in the meniscus: a potential source of pain in osteoarthritis. Ann Rheum Dis. 2011;70:523-9.

25. Bhattacharyya T, Gale D, Dewire P, Totterman S, Gale ME, McLaughlin S, et al. The clinical importance of meniscal tears demonstrated by magnetic resonance imaging in osteoarthritis of the knee. J Bone Joint Surg Am. 2003;85:4-9.

26. Englund M, Niu J, Guermazi A, Roemer FW, Hunter DJ, Lynch JA, et al. Effect of meniscal damage on the development of frequent knee pain, aching, or stiffness. Arthritis Rheum. 2007;56:4048-54.

27. Siemieniuk RAC, Harris IA, Agoritsas T, Poolman RW, Brignardello-Petersen R, Van de Velde $S$, et al. Arthroscopic surgery for degenerative knee arthritis and meniscal tears: a clinical practice guideline. BMJ. 2017;357:j1982.

28. Muratovic D, Cicuttini F, Wluka A, Findlay D, Wang Y, Otto S, et al. Bone marrow lesions detected by specific combination of MRI sequences are associated with severity of osteochondral degeneration. Arthritis Res Ther. 2016;18:54.

29. Lo GH, McAlindon TE, Niu J, Zhang Y, Beals C, Dabrowski C, et al. Bone marrow lesions and joint effusion are strongly and independently associated with weight-bearing pain in knee osteoarthritis: data from the osteoarthritis initiative. Osteoarthr Cartil. 2009;17:1562-9.

30. Hadler NM. Knee pain is the malady--not osteoarthritis. Ann Intern Med. 1992;116:598-9.

31. Okuno Y, Korchi AM, Shinjo T, Kato S, Kaneko T. Midterm clinical outcomes and MR imaging changes after Transcatheter arterial embolization as a treatment for mild to moderate radiographic knee osteoarthritis resistant to conservative treatment. J Vasc Interv Radiol. 2017;28:995-1002.

32. Laslett LL, Dore DA, Quinn SJ, Boon P, Ryan E, Winzenberg TM, et al. Zoledronic acid reduces knee pain and bone marrow lesions over 1 year: a randomised controlled trial. Ann Rheum Dis. 2012;71:1322-8.

33. Meizer R, Radda C, Stolz G, Kotsaris S, Petje G, Krasny C, et al. MRI-controlled analysis of 104 patients with painful bone marrow edema in different joint localizations treated with the prostacyclin analogue iloprost. Wien Klin Wochenschr. 2005;117:278-86.

34. Beard DJ, Pandit H, Ostlere S, Jenkins C, Dodd CA, Murray DW. Pre-operative clinical and radiological assessment of the patellofemoral joint in unicompartmental knee replacement and its influence on outcome. J Bone Joint Surg (Br). 2007;89:1602-7.

35. Hamilton TW, Pandit HG, Maurer DG, Ostlere SJ, Jenkins C, Mellon SJ, et al. Anterior knee pain and evidence of osteoarthritis of the patellofemoral joint should not be considered contraindications to mobile-bearing unicompartmental knee arthroplasty: a 15-year follow-up. Bone Joint J. 2017;99-B:632-9.

36. Grassi A, Compagnoni R, Ferrua P, Zaffagnini S, Berruto M, Samuelsson K, et al. Patellar resurfacing versus patellar retention in primary total knee arthroplasty: a systematic review of overlapping meta-analyses. Knee Surg Sports Traumatol Arthrosc. 2018;26:3206-18.

37. Teel AJ, Esposito JG, Lanting BA, Howard JL, Schemitsch EH. Patellar resurfacing in primary Total knee Arthroplasty: a meta-analysis of randomized controlled trials. J Arthroplast. 2019.

38. Felson DT. Osteoarthritis as a disease of mechanics. Osteoarthr Cartil. 2013; 21:10-5.

39. lijima H, Aoyama T, Ito A, Yamaguchi S, Nagai M, Tajino J, et al. Effects of short-term gentle treadmill walking on subchondral bone in a rat model of instability-induced osteoarthritis. Osteoarthr Cartil. 2015;23:1563-74.

40. Yang $Y$, Wang $Y$, Kong $Y$, Zhang $X$, Bai L. The effects of different frequency treadmill exercise on lipoxin A4 and articular cartilage degeneration in an experimental model of monosodium iodoacetate-induced osteoarthritis in rats. PLoS One. 2017;12:e0179162.

41. Uthman OA, van der Windt DA, Jordan JL, Dziedzic KS, Healey EL, Peat GM, et al. Exercise for lower limb osteoarthritis: systematic review incorporating trial sequential analysis and network meta-analysis. BMJ. 2013;347:f5555.
42. Bannuru RR, Osani MC, Vaysbrot EE, Arden NK, Bennell K, SMA B-Z, et al. OARSI guidelines for the non-surgical management of knee, hip, and polyarticular osteoarthritis. Osteoarthr Cartil. 2019.

43. Fox L, Walsh JC, Morrison TG, OG D, Ruane N, Mitchell C, et al. Cognitive Coping Style and the Effectiveness of Distraction or Sensation-Focused Instructions in Chronic Pain Patients. PLoS One. 2016;11:e0142285.

44. Torres L, Dunlop DD, Peterfy C, Guermazi A, Prasad P, Hayes KW, et al. The relationship between specific tissue lesions and pain severity in persons with knee osteoarthritis. Osteoarthr Cartil. 2006;14:1033-40.

45. Yusuf E, Kortekaas MC, Watt I, Huizinga TW, Kloppenburg M. Do knee abnormalities visualised on MRI explain knee pain in knee osteoarthritis? A systematic review. Ann Rheum Dis. 2011;70:60-7.

46. Davis AM, Badley EM, Beaton DE, Kopec J, Wright JG, Young NL, et al. Rasch analysis of the Western Ontario McMaster (WOMAC) osteoarthritis index: results from community and arthroplasty samples. J Clin Epidemiol. 2003;56:1076-83.

\section{Publisher's Note}

Springer Nature remains neutral with regard to jurisdictional claims in published maps and institutional affiliations.
Ready to submit your research? Choose BMC and benefit from:

- fast, convenient online submission

- thorough peer review by experienced researchers in your field

- rapid publication on acceptance

- support for research data, including large and complex data types

- gold Open Access which fosters wider collaboration and increased citations

- maximum visibility for your research: over $100 \mathrm{M}$ website views per year

At BMC, research is always in progress.

Learn more biomedcentral.com/submissions 